\title{
Remise d'une copie de la facture aux patients selon la LAMal
}

\section{Gabriela Lang}

Avocate, cheffe suppléante de la division Service juridique de la FMH

Correspondance: FMH / Division Service juridique Elfenstrasse 18 CH-3000 Berne 15 Tél. 0313591111 Fax 0313591112 lex[at]fmh.ch
Fin juin 2015, l'Office fédéral de la santé publique (OFSP) a adressé un courrier à la FMH et à d'autres organisations de prestataires en leur demandant de rappeler à leurs membres les dispositions légales suivantes en vigueur concernant le tiers payant et la nécessité de les respecter:

- Art. 42 al. 3 Loi fédérale sur l'assurance-maladie (LAMal), qui prévoit notamment que dans le système du tiers payant, l'assuré reçoit une copie de la facture qui a été adressée à l'assureur.

- Art. 59 al. 4 phrase 1 Ordonnance sur l'assurancemaladie (OAMal), selon laquelle dans le système du tiers payant, le fournisseur de prestations doit remettre à l'assuré la copie de la facture.

- Art. 59 al. 4 phrase 2 OAMal, qui autorise le fournisseur de prestations à convenir avec l'assureur que ce dernier transmettra la copie de la facture à l'assuré.

Les membres de la FMH peuvent consulter l'intégralité du courrier de l'OFSP dans myFMH: myFMH $\rightarrow$ Téléchargements $\rightarrow$ Droit $\rightarrow$ Français $\rightarrow$ Droit des assurances sociales.

\section{Abgabe einer Rechnungskopie an den Patienten gemäss KVG}

\section{Gabriela Lang}

Rechtsanwältin, Stv. Leiterin Abteilung Rechtsdienst der FMH

Korrespondenz:

FMH / Abteilung Rechts

dienst

Elfenstrasse 18

CH-3000 Bern 15

Tel. 0313591111

Fax 0313591112

lex[at]fmh.ch
Ende Juni 2015 hat das Bundesamt für Gesundheit (BAG) die FMH sowie weitere Leistungserbringerverbände angeschrieben, ihre Mitglieder auf die folgenden im Tiers payant geltenden rechtlichen Grundlagen sowie auf die Notwendigkeit deren Einhaltung aufmerksam zu machen:

- Art. 42 Abs. 3 Krankenversicherungsgesetz (KVG), welcher u.a. vorsieht, dass im System des Tiers payant der Versicherte eine Kopie der Rechnung erhält, welche direkt an die Versicherung gegangen ist.

- Art. 59 Abs. 4 Satz 1 Krankenversicherungsverordnung (KVV), welcher festhält, dass im System des
Tiers payant der Leistungserbringer der versicherten Person eine Kopie der Rechnung zukommen zu lassen hat.

- Art. 59 Abs. 4 Satz 2 KVV, wonach der Leistungserbringer mit dem Versicherer vereinbaren kann, dass dieser die Rechnungskopie der versicherten Person zustellt.

Das vollständige Schreiben des BAG können die FMHMitglieder unter myFMH einsehen: myFMH $\rightarrow$ Downloadbereich $\rightarrow$ Recht $\rightarrow$ Deutsch $\rightarrow$ Sozialversicherungsrecht. 